\title{
Diversidade, riqueza e estratificação vertical de espécies de morcegos em um remanescente de Mata Atlântica no Sul do Brasil
}

\author{
Daniel Paulo de Souza Pires * \\ Marta Elena Fabián \\ PPG em Biologia Animal, Departamento de Zoologia \\ Instituto de Biociências, Universidade Federal do Rio Grande do Sul \\ Avenida Bento Gonçalves 9500, Prédio 43435 \\ CEP 91.540-000, Porto Alegre - RS, Brasil \\ * Autor para correspondência \\ pires.daniel@yahoo.com.br
}

Submetido em 09/03/2013

Aceito para publicação em 01/10/2013

\section{Resumo}

Neste estudo, avaliou-se a diversidade, riqueza e a composição das espécies de morcegos no dossel e subbosque de um remanescente de Mata Atlântica no Sul do Brasil, no município de Porto Alegre, Rio Grande do Sul. Entre julho de 2010 e junho de 2011, morcegos foram capturados por meio de 10 redes de neblina, cinco no dossel e cinco no sub-bosque. Foram calculados o índice de Diversidade de Shannon-Wiener (H'), a riqueza esperada (Chao 1 e Jackknife 2) e o índice de constância das espécies para a área como um todo. Aplicou-se o teste Exato de Fisher para verificar se as capturas foram diferentes no dossel e o sub-bosque. Capturaram-se 107 espécimes de quirópteros, 20 indivíduos de cinco espécies no dossel e 87 indivíduos de sete espécies no sub-bosque. O índice de diversidade foi de 1.481e a riqueza esperada de nove (Chao 1) e 10 (Jackknife 2). O índice de constância revelou que Sturnira lilium e Glossophaga soricina são relativamente comuns na área de estudo. A riqueza registrada representa cerca de $22 \%$ das espécies de morcegos listada para o Rio Grande do Sul. A análise de estratificação vertical demonstrou que algumas espécies são mais frequentes no dossel e outras no sub-bosque.

Palavras-chave: Dossel; Floresta estacional; Sub-bosque

\section{Abstract}

Diversity, richness, and vertical stratification of bat species in an Atlantic Forest remnant in the Brazilian southern region. In this study, we evaluated the diversity, richness, and composition of bat species in the canopy and understory of an Atlantic Forest remnant in the Brazilian southern region, in the municipally of Porto Alegre, Rio Grande do Sul. Between July 2010 and June 2011, bats were captured by means of 10 mist nets, 5 in the canopy and 5 in the understory. We calculated the Shannon-Wiener diversity index (H'), the expected richness (Chao 1 and Jackknife 2), and the constancy index of species for the entire area. We applied Fisher's Exact test to check if the catches were different in the canopy and understory. We captured 107 chiropteran specimens, 20 individuals of 5 species in the canopy and 87 individuals of 7 species in the understory. The diversity index was 1,481 and the expected richness was 9 (Chao 1) and 10 (Jackknife 2). The constancy index 
showed that Sturnira lilium and Glossophaga soricina are relatively common in the study area. The registered richness represents about $22 \%$ of bat species listed for the state of Rio Grande do Sul. Vertical stratification analysis showed that some species are more frequent in the canopy and others in the understory.

Key words: Canopy; Seasonal forest; Understory

\section{Introdução}

No Brasil os quirópteros compõem cerca de um terço da fauna de mamíferos e são organismos ativamente inseridos na dinâmica das florestas tropicais (GARDNER, 2007). Morcegos contribuem significativamente para a manutenção das florestas, desempenhando papel importante na dispersão de sementes, na polinização e na predação de insetos (HELVERSEN; WINTER, 2003; JONES; RYDELL, 2003; PATTERSON et al., 2003; FABIÁN et al., 2008). Estes mamíferos são abundantes nas florestas, sendo bons organismos para estudos de diversidade e riqueza de espécies (PATTERSON et al., 2003).

A riqueza e abundância de espécies de morcegos podem variar em função de inúmeros fatores, tais como o grau de conservação do habitat, disponibilidade de recursos alimentares, de água ou de abrigos, a altitude, a latitude, a estratificação e complexidade do habitat e a sazonalidade (KUNZ; KURTA, 1988; PEDRO et al., 1995; REIS et al., 2003; ESBÉRARD; BERGALLO, 2005; DIAS et al., 2008; KALKO et al., 2008; PACHECO et al., 2010).

A estrutura de uma floresta tem muita influência sobre a distribuição vertical de recursos, onde os morcegos se distribuem e ocupam uma alta diversidade de habitats, com espécies que voam e ocupam alturas diferentes da floresta (KALKO et al., 2008). Portanto, estudos que contemplam somente o nível do sub-bosque não são adequados para avaliar a distribuição espaçovertical de morcegos em uma floresta com eficiência, já que a distribuição das espécies varia dentro da estrutura vertical (SIMMONS; VOSS, 1998; KALKO; HANDLEY JR., 2001).

Estudos faunísticos que abrangem os estratos florestais vêm demonstrando diferenças na distribuição e presença de espécies nos diferentes níveis verticais da floresta (GRELLE, 2003; CADEMARTORI et al.,
2008; PREVEDELLO et al., 2008). No entanto, para os morcegos a estratificação ainda é menos evidente do que aquela encontrada nas aves, por exemplo, (PEARSON, 1971; WALTHER, 2002).

No Brasil, vêm crescendo os estudos de riqueza e abundância de espécies de morcegos (FARIA, 2006; DIAS et al., 2008; CARVALHO et al., 2009; BRITTO et al., 2010; CALOURO et al., 2010; ESBÉRARD et al., 2010). No entanto, ainda são poucos os trabalhos publicados que utilizem redes de neblina para estudar padrões de riqueza e abundância que contemplem o estrato vertical da floresta. Com exceção de Weber et al. (2011) e Carvalho et al. (2011) a maioria dos estudos que contemplam mais de um estrato florestal foi realizada em florestas amazônicas (BERNARD, 2001; KALKO; HANDLEY JR., 2001; SAMPAIO et al., 2003; PEREIRA et al., 2010).

Devido à carência de estudos de estratificação vertical de morcegos nas Florestas Estacionais Semideciduais do Rio Grande do Sul, considera-se que o conhecimento existente ainda é insuficiente para compreender com clareza a ecologia deste grupo. O presente estudo teve como objetivos: 1) averiguar a riqueza e diversidade de espécies de quirópteros em um fragmento de Floresta Estacional Semidecidual; 2) verificar a composição das espécies de quirópteros encontradas no dossel e sub-bosque.

\section{Material e Métodos}

O estudo foi realizado na Reserva Ecológica Econsciência, área particular de aproximadamente 142 ha, região de Floresta Estacional Semidecidual localizada no Morro São Pedro (3008'S e $30^{\circ} 12^{\prime}$ S a $51^{\circ} 05^{\prime} \mathrm{O}$ e $\left.51^{\circ} 07^{\prime} \mathrm{O}\right)$, Porto Alegre, Estado do Rio Grande do Sul, Brasil.

O Morro São Pedro (Figura 1) é o maior morro, em área, de Porto Alegre, com 1.259,5 ha, apresentando 
FIGURA 1: Localização do Morro São Pedro, no município de Porto Alegre, Rio Grande do Sul, Brasil.

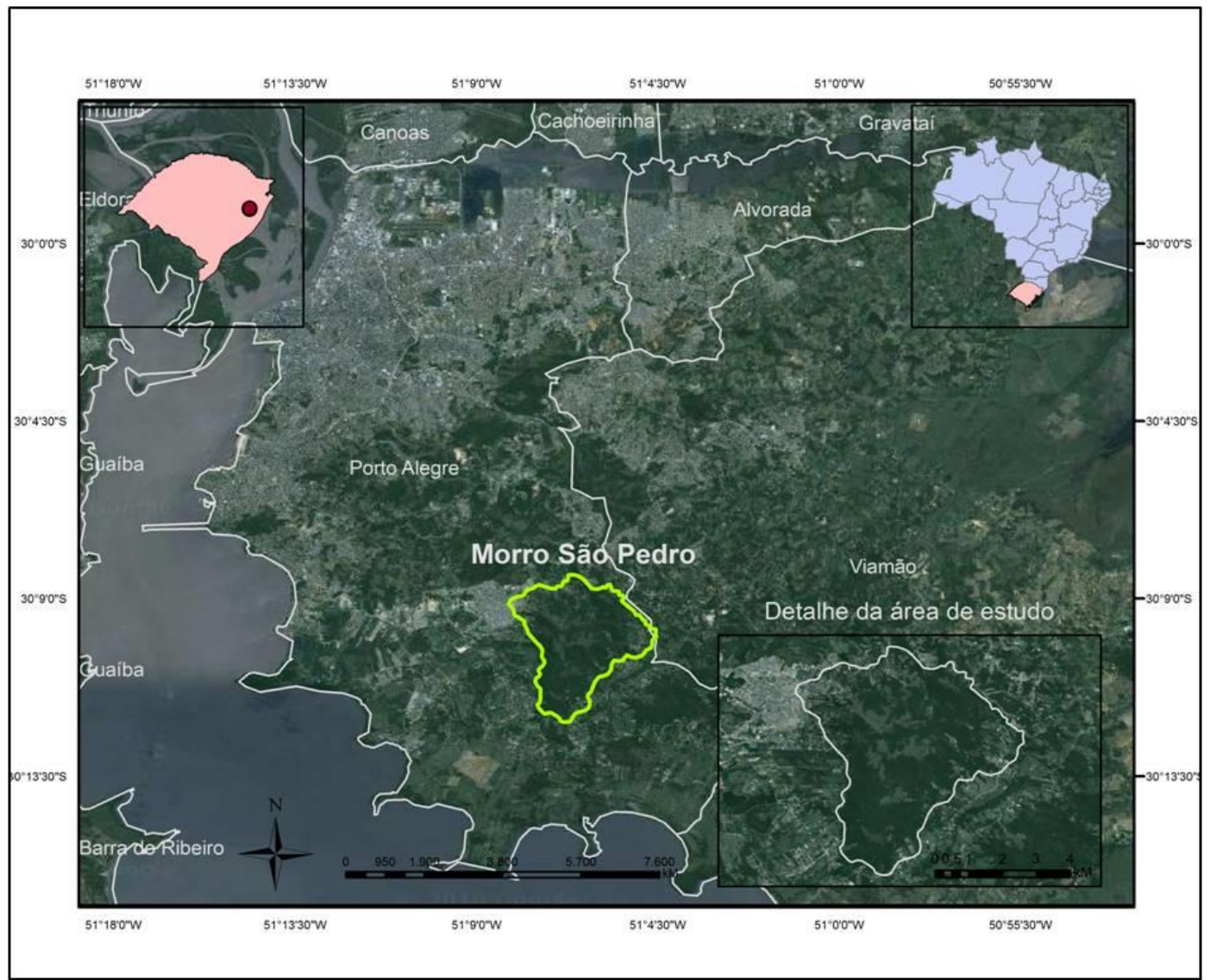

altura máxima de $289 \mathrm{~m}$ em seu ponto culminante. O conjunto de vegetação conservada no Morro São Pedro é um dos maiores maciços remanescentes de Floresta Estacional Semidecidual e campos nativos da capital e um dos mais importantes corredores ecológicos do município, interligando outros fragmentos florestais como o Morro da Extrema, Arroio Lami, Pitinga e Lomba do Pinheiro (RADAM/BRASIL, 1986; PORTO; MELLO, 2006).

A vegetação remanescente dos morros de Porto Alegre distribui-se, de um modo geral, em um mosaico entre mata e campo. A mata apresenta-se mais desenvolvida na base dos morros, principalmente nas vertentes voltadas para sul, onde há árvores emergentes de mais de $15 \mathrm{~m}$ e dossel contínuo variando entre nove e $15 \mathrm{~m}$ de altura. À medida que se avança pelas encostas nota-se a diminuição no porte da mata, em função da modificação das condições edáficas (PORTO; MELLO, 2006).
A área de estudo pertence à bacia hidrográfica do arroio Lami, que tem suas nascentes nos municípios de Viamão e Porto Alegre e drenagem em direção ao lago Guaíba. O arroio Lami tem sua foz na Reserva Biológica do Lami e suas matas ciliares constituem importante corredor ecológico para espécies da flora e fauna silvestre da região sul de Porto Alegre (PORTO; MELLO, 2006).

O clima de Porto Alegre, segundo a classificação de Köppen, corresponde ao subtipo Cfa, possuindo temperatura média anual de $19,4^{\circ} \mathrm{C}$, com máxima absoluta de $37,8^{\circ} \mathrm{C}$ e mínima absoluta de $1,40^{\circ} \mathrm{C}$. A precipitação bem distribuída durante o ano e totais superiores a $1.200 \mathrm{~mm}$ (HASENACK; FERRARO, 2006).

As capturas dos morcegos foram realizadas com redes de neblina armadas em trilhas e clareiras naturais. As redes foram dispostas de maneira a cobrir possíveis 
rotas de voo utilizadas pelos morcegos dentro de cada local selecionado. Dez redes foram utilizadas para o estudo, de metragem $9 \times 3 \mathrm{~m}$, sendo cinco utilizadas na altura do sub-bosque (entre 1,0 e $5 \mathrm{~m}$ ) e cinco estabelecidas na altura do dossel (entre 10 e $15 \mathrm{~m}$ ), abertas desde o início da noite até o início da manhã. As redes foram inspecionadas a cada 30 minutos. A metodologia utilizada para elevar as redes até o dossel foi uma adaptação de Von-Matter (2008) e Carvalho e Fabián (2011). O esforço amostral foi calculado conforme Straube e Bianconi (2002) totalizando um esforço de $116.640 \mathrm{~m}^{2} . \mathrm{h}$, sendo $58.320 \mathrm{~m}^{2} . \mathrm{h}$ em cada estrato.

O estudo foi realizado mensalmente, no período de julho de 2010 a junho de 2011, durante três dias de campo por mês, totalizando 36 noites de coleta. Todas as amostragens foram realizadas na lua nova para evitar o potencial viés de menor capturabilidade causado pela claridade da lua (BREVIGLIERI, 2011).

Os espécimes capturados foram acondicionados em sacos de algodão e posteriormente identificados com a ajuda de chave de identificação e literatura específica (REIS et al., 2007; BARQUEZ; DIAZ, 2009). Os morcegos capturados foram marcados com anilhas de metal colocadas no antebraço direito e em seguida foram soltos na mesma noite de captura distantes a cerca de $100 \mathrm{~m}$ das redes. $\mathrm{O}$ ordenamento taxonômico seguiu a classificação proposta por Gardner (2007).

O cálculo da riqueza estimada foi feito através de dois estimadores de riqueza: 1) Jacknife de segunda ordem, escolhido por permitir a realização de uma estimativa confiável da riqueza mesmo com baixa amostragem (ZAHL, 1977); 2) Chao de primeira ordem, escolhido por ser um estimador simples e poderoso que se baseia na abundância das espécies raras (CHAO, 1984). A suficiência amostral foi verificada a partir do número acumulativo de espécies em função do número de noites amostradas utilizando-se o método de Mao Tau (COLWELL et al., 2004).

Obteve-se o grau de frequência das espécies por meio do cálculo da Constância (C), sendo as espécies classificadas em Comuns ( $\mathrm{C} \geq 50 \%)$, Relativamente Comuns $(25 \% \leq \mathrm{C}<50 \%)$ e Raras na amostragem (C
$<25 \%$ ) (SILVEIRA-NETO et al., 1976; BIANCONI et al., 2004).

Para medir a diversidade de espécies na área de estudo foi utilizado o índice de diversidade de ShannonWiener (H'). Este índice tem sido usado frequentemente por muitos autores, tornando fácil a comparação dos resultados do presente trabalho com outros estudos realizados na Mata Atlântica.

Com o intuito de comparar as diferenças no uso do espaço vertical entre capturas das espécies de morcegos, realizou-se o teste Exato de Fisher, teste indicado para amostras pequenas (VIEIRA, 2010). Atribuiu-se a categoria "dossel" quando a frequência de captura de uma espécie foi maior no dossel (diferença significativa: $\mathrm{p}<0,05$ ). O mesmo procedimento se utilizou para o sub-bosque. Quando a diferença não foi significativa ( $p>0,05)$ atribuiu-se a categoria "sem preferência". O teste foi aplicado para aquelas espécies que apresentaram um número de captura $\geq 6$. A metodologia adotada foi similar à de Kalko e Handley Jr. (2001).

A riqueza de espécies observada no dossel e subbosque foi comparada através de curvas de rarefação (IC 95\%) baseada no número de indivíduos capturados (GOTELLI; COLWELL, 2001).

As estimativas de riqueza foram realizadas através do programa ESTIMATES 8.2 (COLWELL, 2001). O índice de diversidade de Shannon-Wiener ( $\left.\mathrm{H}^{\prime}\right)$, o teste Exato de Fisher, a curva de Mao Tau e as curvas de rarefação foram calculados através do programa Past 3.0 (HAMMER et al., 2001).

\section{Resultados}

A amostragem de quirópteros no Morro São Pedro resultou em 107 capturas de 78 indivíduos distribuídas em nove espécies, representando oito gêneros e três famílias (Tabela 1). Os filostomídeos, com cinco espécies, representaram $95 \%$ das capturas. Os outros $5 \%$ de capturas estão representados por duas espécies da família Vespertilionidae e uma de Molossidae.

Houve rápido incremento de espécies no início da amostragem (primeiras 12 noites), com acréscimos 
TABELA 1: Espécies, número de indivíduos capturados no sub-bosque e dossel, recapturas (entre parênteses), número total de indivíduos capturados e constância dos morcegos capturados entre Julho de 2010 e junho de 2011 no Morro São Pedro, município de Porto Alegre, Rio Grande do Sul, Brasil.

\begin{tabular}{lcccc}
\hline \multicolumn{1}{c}{ Táxon } & Sub-bosque & Dossel & n & IC \\
\hline CHIROPTERA & & & & \\
PHYLLOSTOMIDAE & 3 & 9 & 12 & $\mathrm{R}$ \\
Artibeus lituratus (Olfers, 1818) & 0 & 3 & 3 & $\mathrm{R}$ \\
Artibeus fimbriatus Gray, 1838 & $47(21)$ & 5 & 52 & $\mathrm{RC}$ \\
Sturnira lilium (E. Geoffroy, 1810) & $26(8)$ & 0 & 26 & $\mathrm{RC}$ \\
Glossophaga soricina (Pallas, 1766) & 6 & 1 & 7 & $\mathrm{R}$ \\
Desmodus rotundus (E. Geoffroy, 1810) & & & $\mathrm{R}$ \\
VESPERTILIONIDAE & 2 & 0 & $\mathrm{R}$ \\
Myotis nigricans (Schinz, 1821) & 1 & 0 & 2 & $\mathrm{R}$ \\
Myotis levis (I. Geoffroy, 1824) & 2 & 0 & & $\mathrm{R}$ \\
Histiotus velatus (I. Geoffroy, 1824) & 0 & 2 & $\mathbf{1 0 7}$ & \\
MOLOSSIDAE & $\mathbf{8 7}(\mathbf{2 9})$ & $\mathbf{2 0}$ & \\
Molossus molossus (Pallas, 1766) & & & \\
\hline Totais & & & 2 & \\
\hline
\end{tabular}

Legenda: $\mathrm{n}=$ número total de indivíduos capturados, $\mathrm{IC}=$ Índice de Constância $(\mathrm{RC}=$ Relativamente Comum; $\mathrm{R}=$ raro $)$.

pontuais até o final da amostragem, de forma que, com cerca de $33 \%$ das noites amostradas foi possível obter $67 \%$ da riqueza encontrada nesse estudo. A curva de rarefação espécies-noites pelo método Mao Tau mostrou leve tendência à estabilização em nove espécies. O estimador Chao de primeira ordem estimou nove espécies para área e o Jackknife de segunda ordem estimou 10 espécies (Figura 2). O índice de Shannon-Wiener (H') calculado para a área de estudo foi de 1,481 .

FIGURA 2: Curvas de acumulação de espécies de morcegos capturados entre julho de 2010 e junho de 2011 no Morro São Pedro, município de Porto Alegre, Rio Grande do Sul, Brasil.

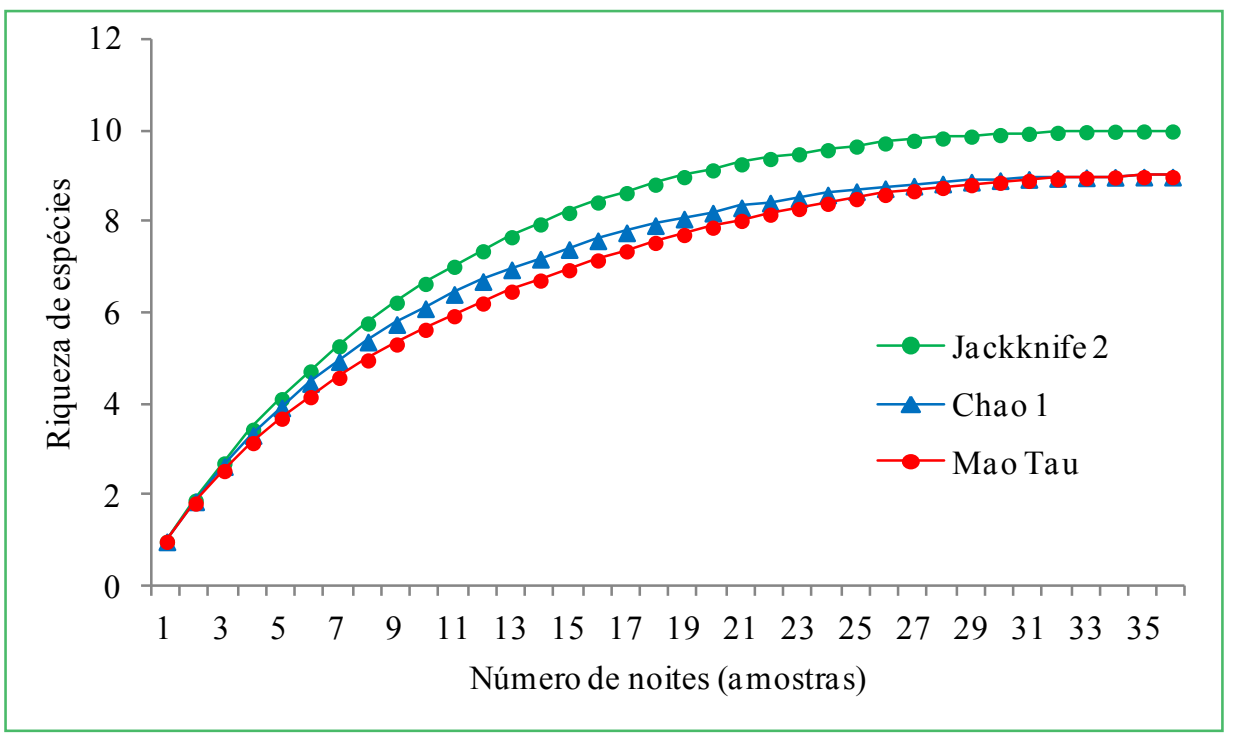


O índice de Constância não revelou espécie comum para a amostra. Sturnira lilium e Glossophaga soricina foram às únicas espécies consideradas relativamente comuns. As demais espécies foram consideradas raras na amostra.

Capturaram-se 87 indivíduos de sete espécies no sub-bosque e 20 indivíduos de cinco espécies no dossel (Tabela 1). Entre os filostomídeos, Artibeus lituratus, Sturnira lilium e Desmodus rorundus foram registrados tanto no sub-bosque quanto no dossel, Glossophaga soricina somente no sub-bosque e Artibeus fimbriatus somente no dossel florestal. Os verpertilionídeos Myotis nigricans, Myotis levis e Histiotus velatus foram capturados exclusivamente no sub-bosque. A espécie Molossus molossus foi registrada somente no dossel.

As capturas foram significativamente diferentes entre dossel e sub-bosques para Sturnira lilium $(\mathrm{p}=$ $0,001)$, A. lituratus $(\mathrm{p}=0,0001)$ e $G$. soricina $(\mathrm{p}=$ $0,0006)$, não significativas para $D$. rotundus $(\mathrm{p}=0,999)$. Sturnira lilium e G. soricina demonstraram preferência, na área de estudo, pelo sub-bosque, enquanto que $A$. lituratus mostrou preferência pelo dossel. Desmodus rotundus não demonstrou preferência.

As curvas resultantes da rarefação por indivíduos indicaram pouca diferença de riqueza específica entre as espécies registradas no sub-bosque e dossel (Figura 3). No ponto de igual esforço amostral (linha tracejada) o dossel apresentou uma riqueza de cinco espécies (IC
95\%: $\pm 1,51$ ), enquanto o sub-bosque apresentou 4,5 espécies (IC 95\%: $\pm 2,735$ ).

\section{Discussão}

A riqueza registrada para o Morro São Pedro compreende cerca de $22 \%$ das espécies de morcegos listada para o Rio Grande do Sul (PASSOS et al., 2010). Para a sub-bacia hidrográfica onde está inserida a área de estudo, o número de espécies registrado compreende cerca de 40\% (PACHECO et al., 2007). Observando-se estes percentuais, considera-se que a área de estudo não está completamente inventariada. Em reforço, as curvas de acúmulo de espécies não mostram tendência definitiva à assíntota, sugerindo que outras espécies podem ser registradas na área de estudo com o aumento das noites de coleta.

O índice de diversidade obtido neste estudo foi superior ao encontrado por Bianconi et al. (2004), equivalente a 1,38. Superior ao encontrado por Gallo et al. (2008), equivalente a 1,36. Inferior ao encontrado por Weber et al. (2011), equivalente a 1,55. Inferior ao encontrado por Dias et al. (2008), equivalente a 2,46. Inferior ao encontrado por Pedro et al. (2001) equivalente a 2,26. Inferior ao encontrado por Faria (2006), equivalente a 1,77. A partir destas comparações, observase que, em áreas de Mata Atlântica, valores do índice de Shannon superiores a 2,0, são registrados apenas em áreas heterogêneas, em bom estado de conservação e

FIGURA 3: Curvas de rarefação representando o número esperado de espécies de morcegos para o dossel e o sub-bosque. Barras verticais representam o intervalo de confiança (95\%).

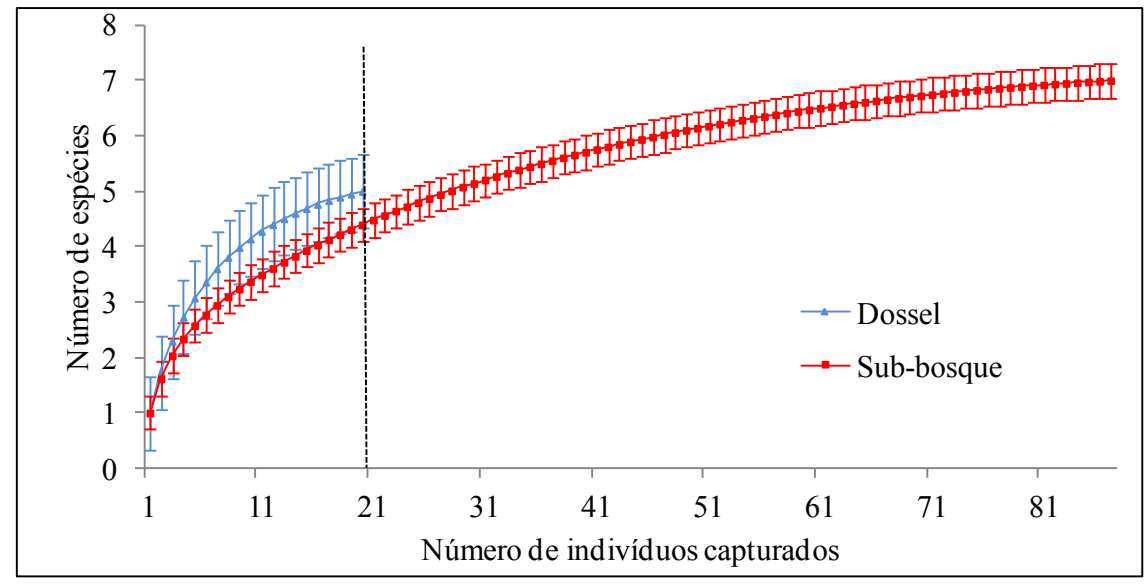


localizadas em latitudes inferiores a $20^{\circ} \mathrm{S}$ (PEDRO et al., 2001; PATTERSON et al., 2003; DIAS et al., 2008).

A predominância de filostomídeos parece ser um padrão em estudos neotropicais, sendo a família com maior riqueza e abundância nos resultados de pesquisas realizadas na região sul do país que utilizam redes de neblina (BIANCONI et al., 2004; CARVALHO et al., 2009; ORTÊNCIO-FILHO; REIS, 2009; WEBER et al., 2011). Tal predominância, tanto em número de indivíduos quanto em número de espécies, pode-se justificar pelo fato de ser a família mais fácil de capturar através das redes de neblina (KUNZ; KURTA, 1988).

Em relação aos vespertilionídeos e molossídeos, os resultados encontrados neste trabalho assemelham-se aos de outros estudos no sul do Brasil que utilizam redes de neblina, em que os morcegos insetívoros são pouco capturados (BIANCONI et al., 2004; CARVALHO et al., 2009; WEBER et al., 2011). Isso, possivelmente, se deve ao fato de os morcegos insetívoros possuírem uma capacidade mais aguçada de detectar as redes através da eco-localização (KUNZ; KURTA, 1988).

Apesar do índice de Constância não apontar espécie comum para a área de estudo, S. lilium foi numericamente a espécie predominante, o que parece ser padrão em alguns estudos neotropicais (FALCÃO et al., 2003; REIS et al., 2006; BERNARDI; PASSOS, 2012), sendo uma das espécies mais abundantes na região Neotropical. Entretanto, em boa parte de trabalhos realizados em Mata Atlântica, a espécie predominante pode variar entre $A$. lituratus, A. fimbriatus e $S$. lilium (BIANCONI et al., 2004; ORTÊNCIO-FILHO et al., 2005; GALLO et al., 2008; ORTÊNCIO-FILHO; REIS, 2009; BRITTO et al., 2010). Mesmo assim, é consenso que estes frugívoros são os mais representativos em inventários florestais no sul do Brasil (RUI; FÁBIAN, 1997; BIANCONI et al., 2004; CARVALHO et al., 2009; WEBER et al., 2011).

A despeito de ser a segunda espécie com mais registros nesse estudo, G. soricina é uma das espécies menos representativas ou é até mesmo ausente em inventários realizados em Mata Atlântica (REIS et al., 2003; BIANCONI et al., 2004; GALLO et al., 2008; DIAS et al., 2008; ORTÊNCIO-FILHO; REIS, 2009;
BRITTO et al., 2010). Em Floresta Estacional Decidual no Rio Grande do Sul, Weber et al., (2011) obtiveram poucos registros dessa espécie. A presença elevada de G. soricina nesse estudo pode ser explicada em parte pela presença de algumas plantações de banana ( $M u s a$ sp.) próximas ao local de amostragem. De acordo com Fabián et al., (2008) G. soricina pode alimentar-se tanto do fruto quanto do néctar das bananeiras.

A presença de $D$. rotundus em alguns dos inventários realizados no sul do Brasil está associada à existência de cavernas bem como à presença de animais de criação como suínos, equinos e principalmente bovinos (BIANCONI et al., 2004; ORTÊNCIO-FILHO; REIS, 2009). Embora a Reserva Ecológica do Econsciência não tenha atividades agropecuárias, no entorno da região existem muitas fazendas com este tipo de atividade. Além disso, é fácil encontrar pequenas cavernas nas encostas do Morro São Pedro. Estas características, em conjunto, podem fornecer alimento e abrigo para o morcego hematófago.

Com relação a estratificação vertical, os padrões gerais de riqueza e abundância observados neste estudo assemelham-se a outros estudos neotropicais onde o dossel e os estratos inferiores são amostrados em conjunto (BERNARD, 2001; KALKO; HANDLEY JR., 2001; PEREIRA et al., 2010).

As redes de sub-bosque capturaram mais espécies, contudo, assim como em outros estudos de estratificação vertical, a combinação da amostragem no sub-bosque e dossel, foi mais efetiva do que os dois métodos isoladamente, demonstrando que essa combinação de redes em níveis diferentes dos estratos, aumenta a efetividade de inventários locais (BERNARD, 2001; KALKO; HANDLEY JR., 2001; PEREIRA et al., 2010).

No trabalho de Kalko e Handley Jr. (2001), realizado na Amazônia, A. lituratus apresentou a maior parte das capturas no dossel. Os resultados de Weber et al. (2011) assemelham-se muito aos encontrados nesse estudo, onde $A$. fimbriatus e $A$. lituratus parecem utilizar os dois estratos com relativa frequência. Em outros trabalhos a frequência de captura de $A$. lituratus é melhor distribuída entre os estratos, contudo sendo sempre superior no dossel (BERNARD, 2001; PEREIRA 
et al., 2010). Em contrapartida, morcegos deste gênero são muito frequentemente capturados em estudos que abrangem somente o estrato inferior da vegetação (RUI; FABIAN, 1997; BIANCONI et al., 2004; BRITTO et al., 2010; WEBER et al., 2011). Esse padrão de uso dos dois estratos pode ser explicado pelos seus hábitos de se alimentarem primariamente de frutos localizados no dossel e de utilizar o estrato inferior como rota de vôo em seus deslocamentos (KALKO et al., 2008). Porém, assim como na maioria dos trabalhos de estratificação vertical, os resultados desse estudo apontam o dossel como extrato preferencial de $A$. lituratus e $A$. fimbriatus (BERNARD, 2001; KALKO; HANDLEY JR., 2001; PEREIRA et al., 2010).

A comparação deste estudo com outros trabalhos reafirma a preferência de $S$. lilium pelo sub-bosque (BERNARD, 2001, PEREIRA et al., 2010; WEBER et al., 2011). Neste trabalho, S. lilium foi a espécie mais influenciou na diferença de abundância entre os estratos. Sturnira lilium representa cerca de 55\% dos indivíduos amostrados no sub-bosque, atribuindo a este estrato um número de capturas quatro vezes maior que no dossel. Em reforço todas as recapturas dessa espécie foram no sub-bosque. A preferência desta espécie pelo subbosque está relacionada, principalmente, ao seu hábito de consumir mais frequentemente plantas encontradas nesse estrato (FABIÁN et al., 2008; MELLO, 2009).

Os resultados desse trabalho sugerem que G. soricina possui preferência pelo sub-bosque, corroborando com os resultados de Weber et al. (2011). No entanto, Pereira et al. (2010), em estudo realizado na Amazônia, demonstraram que esta espécie é flexível em relação ao uso do espaço, não exibindo preferência. O que pode explicar essa variação vertical da espécie são seus hábitos alimentares nectarívoros/oportunísticos (MELLO, 2009). Provavelmente, G. soricina forrageia em diferentes estratos de acordo com a disponibilidade de alimento no espaço vertical da floresta (GIANNINI, 1999).

No presente trabalho $D$. rotundus apresentou maior parte de suas capturas no sub-bosque, não apresentando diferenças significativas entre capturas nos estratos. Considera-se que o $n$ amostral dessa espécie, neste trabalho, foi muito baixo para determinar o habitat de voo preferencial desse morcego. Apesar disso, é consenso em trabalhos de estratificação vertical que este morcego hematófago prefere voar no sub-bosque (BERNARD, 2001; KALKO; HANDLEY JR., 2001; PEREIRA et al., 2010). O padrão de uso do habitat por parte dessa espécie está ligado a sua dieta hematófaga, que se constitui de médios e grandes mamíferos (REIS et al., 2007).

Como evidenciado por outros autores (BERNARD, 2001; KALKO; HANDLEY JR., 2001; PEREIRA et al., 2010), para aquelas espécies com poucos registros, é difícil traçar um perfil do uso do estrato vertical. No entanto, quando se avaliam alguns trabalhos de atividade morcegos insetívoros da família Vespertilionidae, há uma tendência de captura nos estratos superiores (HAYES; GRUVER, 2000; ADAMS et al., 2009).

Molossídeos são conhecidos por serem abundantes nas regiões periurbanas (PACHECO et al., 2010). Usualmente realizam vôos a grandes alturas acima do dossel e bem distantes das redes de neblina (KALKO et al., 1996; 2008). Membros dessa família são fracamente amostrados em estudos de estratificação vertical em florestas que utilizam redes de neblina (BERNARD, 2001; SAMPAIO et al., 2010). Neste estudo, dois indivíduos de $M$. molossus foram capturados nas redes posicionadas no dossel (15 $\mathrm{m}$ de altura), confirmando a ideia de que estes animais voam a grandes alturas e são de difícil captura (KALKO; HANDLEY JR., 2001).

A despeito da composição de espécies entre os estratos registrada nesse trabalho ser distinta, a riqueza especifica não foi muito diferente, o que parece ser comum em trabalhos de estratificação vertical, onde, apesar de certas espécies terem preferência por um tipo de estrato florestal, o número de espécies registrados em cada ambiente varia muito pouco (BERNARD, 2001; KALKO; HANDLEY JR., 2001; PEREIRA et al., 2010).

Os resultados apresentados neste trabalho demonstram que o padrão de riqueza e diversidade de espécies é muito semelhante a outros estudos realizados no Brasil, principalmente aqueles realizados em áreas antropizadas. Apesar da baixa diversidade de espécies, o morro São Pedro abriga uma parcela significativa das espécies de morcegos esperadas para a região. Além 
disso, foi possível demonstrar que algumas espécies de morcegos encontradas no Morro São Pedro possuem associação importante com o estrato vertical. Apesar de não ser possível avaliar tal associação para todas as espécies de morcegos registradas nesse estudo, sendo evidente que a estrutura vertical da floresta pode ser um fator que regula a riqueza e abundância das espécies de morcegos da região. Sendo este o maior remanescente de Mata Atlântica do município de Porto Alegre, é provável que a área de estudo se enquadre entre os fragmentos florestais mais importantes para o estabelecimento de espécies de quirópteros dentro do município.

\section{Agradecimentos}

Agradecemos a Coordenação de Aperfeiçoamento de Pessoal de Nível Pessoal (CAPES) pela bolsa de estudos (Mestrado), ao Programa de Pós-Graduação em Biologia Animal da URFGS pelo apoio financeiro e científico e a Felipe Viana pelo grande apoio, incentivo e ajuda em campo.

\section{Referências}

ADAMS, M. D.; LAW, B. S.; FRENCH, K. O. Vegetation structure influences the vertical stratification of open and edge space aerial-foraging bats in harvested forests. Forest Ecology and Management, Amsterdam, v. 258, p. 2090-2100, 2009.

BARQUEZ R. M.; DÍAZ M. M. Los murciélagos de Argentina: clave de identificación. Tucumán: PCMA (Programa de Conservación de los Murciélagos de Argentina), 2009. 84 p.

BERNARD, E. Vertical stratification of bat communities in primary forest of Central Amazon, Brazil. Journal of Tropical Ecology, Winchelsea, v. 17, p. 115-126, 2001.

BERNARDI, I.; PASSOS, F. C. Estrutura de comunidade de morcego em relictos de floresta estacional decidual no Sul do Brasil. Mastozoologia Neotropical, Mendonza, v. 19, n. 1, p. 9-20, 2012.

BIANCONI, G. V.; MIKICH, S. B.; PEDRO, W. A. Diversidade de morcegos (Mammalia, Chiroptera) em remanescentes florestais do município de Fênix, noroeste do Paraná, Brasil. Revista Brasileira de Zoologia, Curitiba, v. 21, n. 4, p. 943-954, 2004.

BREVIGLIERI, C. P. B. Influência do dossel na atividade de morcegos (Chiroptera: Phyllostomidae) em três fragmentos no estado de São Paulo. Chiroptera Neotropical, Brasília, v. 17, n. 1, p. 817-825, 2011.

BRITTO, J. E. C.; GAZARINI, J.; ZAWADZKI, C. H. 2010. Abundância e frugívoria da quiropterofauna (Mammalia, Chiroptera) de um fragmento no noroeste do estado do Paraná, Brasil. Acta Scientiarum Biological Sciences, Maringá, v. 32, n. 3, p. 265-271, 2010.
CADEMARTORI, C. V.; MARQUES, R. V.; PACHECO, S. M. Estratificação vertical no uso do espaço por pequenos mamíferos (Rodentia, Sigmodontinae) em área de Floresta Ombrófila Mista, RS, Brasil. Revista Brasileira de Zoociências, Juiz de Fora, v. 10, n. 3, p. 187-194, 2008.

CAlOURO, A. M.; SANTOS, F. G. A.; FAUSTINO, C. L.; SOUZA, S. F.; LAGUE, B. M.; MARCIENTE, R.; SANTOS, G. J. L. Riqueza e abundância de morcegos capturados na borda e no interior de um fragmento florestal do estado do Acre, Brasil. Biotemas, Florianópolis, v. 23, n. 4, p. 109-117, 2010.

CARVALHO, E. D. C.; FREITAS, L. N.; FREITAS, G. F.; LUZ, J. L.; COSTA, L. M.; ESBÉRARD, E. L. Efeito da chuva na captura de morcegos em uma ilha da costa sul do Rio de Janeiro, Brasil. Chiroptera Neotropical, Brasília, v. 17, n. 1, p. 808-816, 2011.

CARVALHO, F.; FABIÁN, M. E. Método para instalação de redes de neblina em dosséis florestais para amostragem de morcegos (Mammalia; Chiroptera). Chiroptera Neotropical, Brasília, v. 17, n. 1, p. 795-802, 2011

CARVALHO, F.; ZOCCHE, J. J.; MENDONÇA, R. Á. Morcegos (Mammalia, Chiroptera) em restinga no município de Jaguaruna, sul de Santa Catarina, Brasil. Biotemas, Florianópolis, v. 22, n. 3, p. 193-201, 2009.

CHAO, A. Non-parametric estimation of number of classes in a population. Scandinavian Journal of Statistics, Oxford, v. 11, p. 265-270, 1984

COLWELL, R. K. Estimates: statistical estimation of species richness and shared species from samples. Version 8.0.b1. User's Guide and application. Disponível em: $<$ http://viceroy.eeb.uconn. edu/estimates $>$. Acesso em: 22 ago. 2011.

COLWELL, R. K.; MAO, C. X.; CHANG, J. Interpolating, extrapolating, and comparing incidence-based species accumulation curves. Ecology, Ithaca, v. 85, n. 10, p. 2717-2727, 2004.

DIAS, D.; ESBÉRARD, C. E. L.; PERACCHI, A. Riqueza, diversidade de espécies e variações altitudinal de morcegos na Reserva Biológica do Tiguá, estado do Rio de Janeiro, Brasil (Mammalia, Chiroptera). In: REIS, N. R.; PERACCHI, A. L.; SANTOS, G. A. S. D. (Ed.). Ecologia de morcegos. Londrina: Technical Books, 2008. p. 125-142.

ESBÉRARD, C. E. L.; BERGALLO, H. G. Coletar morcegos por seis ou doze horas a cada noite? Revista Brasileira de Zoologia, Curitiba, v. 22, n. 4, p. 1095-1098, 2005.

ESBÉRARD, C. E. L.; BAPTISTA, M.; COSTA, L. M.; LUZ, J.; LOURENÇO, E. C. Morcegos de Paraíso do Tobias, Miracema, Rio de Janeiro. Biota Neotropica, Campinas, v. 10, n. 4, p. 249$255,2010$.

FALCÃO, C. F.; REBELO, F. V.; TALAMONI, A. S. Structure of a bat assemblage (Mammalia, Chiroptera) in Serra do Caraça Reserve, South-East Brazil. Revista Brasileira de Zoologia, Curitiba, v. 20, n. 2, p. 347-350, 2003.

FABIÁN, M. E.; RUI, A. M.; WAECHTER, J. L. Plantas utilizadas como alimento por morcegos (Chiroptera, Phyllostomidade), no Brasil. In: REIS, N. R.; PERACCHI, A. L.; SANTOS, G. A. S. D. (Ed.). Ecologia de morcegos. Londrina: Technical Books, 2008. p. 51-70.

FARIA. D. Phyllostomid bats of a fragmented landscape in the north-eastern Atlantic forest, Brazil. Journal of Tropical Ecology, Winchelsea, v. 22, p. 531-542, 2006. 
GALLO, P. H.; REIS, N. R.; ANDRADE, F. R.; ALMEIDA, I. G. 2008. Morcegos (Mammalia, Chiroptera) encontrados em fragmentos de mata nativa e reflorestamento no Município de Rancho Alegre, Paraná. In: REIS, N. R.; PERACCHI, A. L.; SANTOS, G. A. S. D. (Ed.). Ecologia de morcegos. Londrina: Technical Books, 2008. p. 97-107.

GARDNER, A. L. Mammals of South America: marsupials, xenarthrans, shrews, and bats. Chicago: The University of Chicago Press, 2007. 669 p.

GIANNINI, N. Selection of diet and elevation by sympatric species of Sturnira in an Andean rainforest. Journal of Mammalogy, Lawrence, v. 80, p. 1186-1195, 1999.

GOTELLI, N. J.; COLWELL, R. K. Quantifying biodiversity: procedures and pitfalls in the measurement and comparison of species richness. Ecology Letters, Montpellier, v. 4, p. 379-391, 2001.

GRELLE, C. E. V. Forest structure and vertical stratification of small mammals in a secondary Atlantic Forest, Southeastern Brazil. Studies on Neotropical Fauna and Environment, London, v. 38, p. 81-85, 2003.

HAMMER, O.; HARPER, D. A. T.; RYAN, P. D. 2001. PAST. Paleontological Statistics software package for education and data analysis. Palaeontologia Electronica, Oslo, v. 41, n. 1, p. 1-9, 2001.

HASENACK, H.; FERRARO, L. W. Clima urbano: ilhas de calor e ventos fortes na selva de pedra. In: MENEGAT, R.; PORTO, M. L.; CARRARO, C. C.; FERNANDES, L. A. D. (Coord.). Atlas ambiental de Porto Alegre. 3. ed. Porto Alegre: Universidade Federal do Rio Grande do Sul, 2006. p. 149-150.

HAYES, J. P.; J. C. GRUVER. 2000. Vertical stratification of bat activity in an oldgrowth forest in western Washington. Northwest Science, Washington, v. 74, p. 102-108, 2000.

HELVERSEN, O.; WINTER, Y. 2003. Glossophaginae bats and their flowers: costs and benefits for plant and pollinators. In: KUNZ, T. H.; FENTON, M. B. (Ed.). Bat Ecology. Chicago: University Chicago Press, 2003. p. 346-389.

JONES, G.; RYDELL, J. 2003. Attack and defense: Interactions between echolocating bats and their insect prey. In: KUNZ, T. H.; FENTON, M. B. (Ed.). Bat Ecology. Chicago: University Chicago Press, 2003. p. 536-579.

KALKO, E. K. V.; HANDLEY JR., C. O. Neotropical bats in the canopy: diversity, community structure and implications for conservations. Plant Ecology, Dordrecht, v. 153, p. 319-333, 2001.

KALKO, E. K. V.; HANDLEY JR., C. O.; HANDLEY, D. Organization, diversity, and long-term dynamics of a neotropical bat community. In: CODY, M.; SMALLWOOD, J. (Ed.). Long term studies in vertebrate communities. San Diego: Academica Press, 1996. p. 503-551.

KALKO, E. K. V.; VILLEGAS, S. E.; SCHIMIDT, M.; WEGMANN, M. ; MEYER, C. F. J. Flying high - assessing the use of the aerosphere by bats. Integrative and Comparative Biology, Oxford, v. 48, p. 60-73, 2008.

KUNZ, T. H.; KURTA, A. Capture methods and holding devices: In: KUNZ, T. H. (Ed.). Ecological and behavioral methods for the study of bats. Washington: Smithsonian Institution Press, 1988. p. 1-28.

MELLO, M. A. R. Temporal variation in the organization of a Neotropical assemblage of leaf-nosed bats (Chiroptera: Phyllostomidae). Acta Oecologica, New York, v. 35, p. 280-286, 2009.

ORTÊNCIO-FILHO, H.; REIS, N. R. Species richness and abundance of bats in fragments of the Stational Semidecidual Forest, upper Paraná River, Southern Brazil. Brazilian Journal of Biology, São Carlos, v. 69, n. 2, p. 727-734, 2009.

PACHECO, S. M.; SEKIAMA, M. L.; OLIVEIRA, K. P. A; QUINTELA, F.; WEBER, M. M.; MARQUES, R. V.; GEIGER, D.; SILVEIRA, D. D. Biogeografia de quirópteros da Região Sul. Ciência \& Ambiente, Santa Maria, v. 35, p. 181-202, 2007.

PACHECO, S. M.; SODRÉ, M.; GAMA, A. R.; BREDT, A.; CAVALLINI-SANCHES, E. M.; MARQUES, R. V.; GUIMARÂES, M. M.; BIANCONI, G. Morcegos urbanos: status do conhecimento e plano de ação de conservação no Brasil. Chiroptera Neotropical, Brasília, v. 16, n. 1, p. 630-647, 2010.

PASSOS, F. C.; MIRANDA, J. M. D.; BERNARDI, I. P.; KAKUOLIVEIRA, N. Y.; MUNSTER, L. C. 2010. Morcegos da Região sul do Brasil: análise comparativa da riqueza de espécies, novos registros e atualizações nomenclaturais (Mammalia, Chiroptera). Iheringia - Série Zoologia, Porto Alegre, v. 100, n. 1, p. 25-34, 2010.

PATTERSON, B. D.; WILliG, M. R.; STEVENS, R. D. Trophic strategies, niche partitioning, and patterns of ecological organization. In: KUNZ, T. H.; FENTON, M. B. (Ed.). Bat Ecology. Chicago: University Chicago Press, 2003. p. 536-579.

PEARSON, D. L. Vertical stratification of birds in a Tropical dry forest. The Condor, Albuquerque, v. 73, p. 46-55, 1971.

PEDRO, W. A.; GERALDES, M. P.; LOPEZ, G. G.; ALHO, C. J. R. Fragmentação de hábitat e a estrutura de uma taxocenose de morcegos em São Paulo (Brasil). Chiroptera Neotropical, Brasília, v. 1, n. 1, p. 4-6, 1995.

PEREIRA, M. J. R.; MARQUES, J. T.; PALMERIM, J. M. Vertical stratification of bat assemblages in flooded and unflooded Amazonian forests. Current Zoology, Beijin, v. 56, n. 4, p. 469478, 2010 .

PORTO, M. L.; MELLO, R. Mapa da vegetação natural atual. In: MENEGAT, R.; PORTO, M. L.; CARRARO, C. C.; FERNANDES, L. A. D. (Coord.). Atlas ambiental de Porto Alegre. 3. ed. Porto Alegre: Universidade Federal do Rio Grande do Sul, 2006. p. 5358.

PREVEDELLO, J. A.; FERREIRA, P.; PAPI, B. S.; LORETTO, D. ; VIEIRA, M. V. Uso do espaço vertical por pequenos mamíferos no parque nacional serra dos órgãos, Rio de Janeiro: um estudo de 10 anos utilizando três métodos de amostragem. Espaço \& Geografia, Brasília, v. 11, n. 1, p. 35-58, 2008.

RADAM BRASIL. Levantamento de Recursos Naturais. Vol 33. Folha SH. 22 Porto Alegre e Parte das Folhas SH. 21 Uruguaiana e SI. 22 Lagoa Mirim. Rio de Janeiro: IBGE, 1986. 791 p.

REIS, N. R.; BARBIERI, M. L. S.; LIMA, I. P. L.; PERACCHI, A. L. O que é melhor para manter a riqueza de espécies de morcegos (Mammalia, Chiroptera): um fragmento florestal grande ou vários fragmentos de pequeno tamanho? Revista Brasileira de Zoologia, Curitiba, v. 20, n. 2, p. 225-230, 2003.

REIS, N. R.; PERACCHI, A. L.; LIMA, I. P.; PEDRO, W. A. Riqueza de espécies de morcegos (Mammalia, Chiroptera) em dois diferentes habitats, na região centro-sul do Paraná, sul do Brasil. 
Revista Brasileira de Zoologia, Curitiba, v. 23, n. 3, p. 813-816, 2006.

REIS, N. R.; PERACCHI, A. L.; PEDRO, W. A.; LIMA, I. P. Morcegos do Brasil. Londrina: Nélio R. dos Reis, 2007. 253 p.

RUI, A. M.; FABIÁN, M. E. Quiropteros de la familia Phyllostomidae (Mammalia, Chiroptera) en selvas del estado de Rio Grande do Sul, Brasil. Chiroptera Neotropical, Brasília, v. 2, n. 3, p. 75-77, 1997.

SAMPAIO, E. M.; KALKO, E. K. V.; BERNARD, E.; RODRIGUÉZ-HERRERA, B.; HANDLEY, C. O. 2003. A biodiversity assessment of bats (Chiroptera) in a Tropical Lowland Rainforest of Central Amazonia, including methodological and conservation considerations. Studies on Neotropical Fauna and Environment, London, v. 38, p. 17-31, 2003.

SILVEIRA-NETO, S. O.; NAKANO, D.; NOVA, N. A. V. 1976. Manual de ecologia dos insetos. São Paulo: Ceres, 1976. 419 p.

SIMMONS, N. B. 2005. Order Chiroptera. In: WILSON, D. E.; REEDER, D. M. (Ed.). Mammal species of the world: a taxonomic and geographic reference. 3 ed. Baltimore: The Johns Hopkins University Press, 2005. p. 312-529.
SIMMONS, N.; VOSS, R. S. The mammals of Paracou, French Guiana: a neotropical lowland forest fauna. Part I. Bats. Bulletin of the American Museum of Natural History, New York, v. 237, p. 1-219, 1998.

STRAUBE, F. C.; BIANCONI, G. V. Sobre a grandeza e a unidade utilizada para estimar esforço de captura com utilização de redesde-neblina. Chiroptera Neotropical, Brasília, v. 8, n. 1-2, p. 150$152,2002$.

VON-MATTER, S. Amostragem com rede de neblina em dosséis florestais. Ornithologia, Palmas, v. 3, n. 1, p. 43-63, 2008.

VIEIRA, S. Bioestatística, tópicos avançados. 3 ed. Rio de Janeiro: Elsevier, 2010. 278 p.

WALTHER, B. A. Vertical stratification and use of vegetation and light habitats by Neotropical forest birds. Journal für Ornithologie, Berlin, v. 143, p. 64-81, 2002.

WEBER, M. M.; ARRUDA, J. L. S.; AZAMBUJA, B. O.; CAMILOTTI, V. L.; CACERES, N. C. Resources partitioning in a fruit bat community of the southern Atlantic Forest, Brazil. Mammalia, Paris, v. 75, p. 217-225, 2011.

ZAHL S. Jackknifing an index of diversity. Ecology, New York, v. 58, p. 907-913, 1977. 